
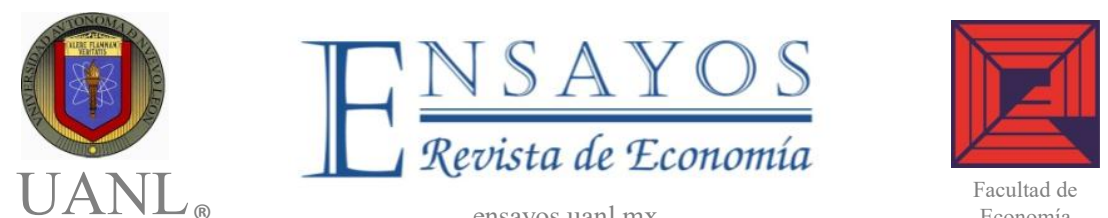

Facultad de

ensayos.uanl.mx

Economía

UNIVERSIDAD AUTÓNOMA DE NUEVO LEÓN $\square$ FACULTAD DE ECONOMIA $\square$ CENTRO DE INVESTIGACIONES ECONÓMICAS

\title{
Sostenibilidad del gasto público: Cobertura y financiamiento de enfermedades crónicas en México
}

\section{Public Spending Sustainability: Coverage and Financing of Chronic Diseases in Mexico}

\section{Alejandra Macías Sánchez* Héctor Juan Villarreal Páez}

\begin{tabular}{|c|c|}
\hline $\begin{array}{l}\text { Información del } \\
\text { artículo }\end{array}$ & Resumen \\
\hline $\begin{array}{l}\text { Recibido: } \\
17 \text { febrero } 2017\end{array}$ & \multirow{10}{*}{$\begin{array}{l}\text { Este artículo analiza la sostenibilidad del gasto } \\
\text { público en salud, desde la perspectiva del costo de } \\
\text { las principales enfermedades crónicas que padece } \\
\text { la población mexicana, y que debe cubrir el } \\
\text { gobierno federal. Asimismo, pretende abrir la } \\
\text { discusión sobre la cobertura efectiva universal de } \\
\text { salud en México, a través de una herramienta de } \\
\text { microsimulación que permita presentar y evaluar } \\
\text { diferentes escenarios en cuanto a su costo } \\
\text { financiero, para poder hacer propuestas de } \\
\text { financiamiento sostenible, principalmente porque } \\
\text { se están enfrentando cambios demográficos y } \\
\text { epidemiológicos que conllevan aumentos en los } \\
\text { costos de atención médica. }\end{array}$} \\
\hline $\begin{array}{l}\text { Aceptado: } \\
21 \text { febrero } 2018\end{array}$ & \\
\hline $\begin{array}{l}\text { Clasificación JEL: } \\
\text { C15; H51; J11 }\end{array}$ & \\
\hline Palabras c & \\
\hline & \\
\hline Modelos de simulación; & \\
\hline & \\
\hline $\begin{array}{l}\text { Transición den } \\
\text { Cambios }\end{array}$ & \\
\hline & \\
\hline Sostenibilidad Fiscal & \\
\hline
\end{tabular}

* School of Government and Public Transformation- Tecnológico de Monterrey. Dirección: Carlos Lazo No. 100, Colonia Santa Fe, C.P. 01389, Álvaro Obregón, México, D.F. escueladegobierno.itesm.mx. a01120992@itesm.mx; alejandramaci@ciep.mx

School of Government and Public Transformation- Tecnológico de Monterrey. hjvp@itesm.mx 


\begin{tabular}{lll}
\hline Article information & & Abstract \\
\cline { 1 - 1 } $\begin{array}{l}\text { Received } \\
\text { 17 February } 2017\end{array}$ & $\begin{array}{l}\text { This article analyzes the sustainability of public } \\
\text { health expenditures from the cost perspective of } \\
\text { the principle chronic diseases that the Mexican }\end{array}$ \\
Accepted & population suffers and the Mexican Government \\
21 February 2018 & would finance. Also, it pretends to contribute to \\
JEL Classification: & the discussion of universal effective health \\
C15; H51; J11 & coverage in Mexico by providing a micro \\
Keywords: & simulation tool which would evaluate different \\
Public health spending; & scenarios about financial costs of universal \\
Simulation models; & coverage. As a result, fiscally sustainable \\
Chronic diseases; & proposals can be made based on this \\
$\begin{array}{l}\text { Demographic } \\
\text { transition.; }\end{array}$ & microsimulation tool that consider the \\
Epidemiological & demographic and epidemiological changes that \\
changes; Fiscal & translate into costs increases. \\
sustainability &
\end{tabular}

\section{Introducción}

El objetivo de este artículo es tratar de explicar el monto que el gobierno gasta y gastará en salud, bajo distintos escenarios, a través de un modelo de simulación basado en la atención de las enfermedades crónicas no transmisibles, que presentan en mayor medida los pobladores de México (simulador del sistema de salud), las cuales tienen un alto costo de tratamiento. El modelo de microsimulación contiene información de tres enfermedades no transmisibles: diabetes, hipertensión y depresión. Estas enfermedades se presentan con mayor frecuencia en las instituciones de salud pública. Además, se cuenta con información de diagnóstico y tratamiento de ellas a partir de la ENSANUT 2012. Cabe mencionar que en 2016, IMSS e ISSSTE gastaron en conjunto alrededor de $95 \mathrm{mmdp}$ de sus presupuestos en atender diabetes, hipertensión, insuficiencia renal y cáncer cérvico uterino y de próstata. Lo anterior equivale al $31.4 \%$ del presupuesto total destinado a estas instituciones para salud. Para ambas instituciones, es consistente que la fecuencia en consultas y egresos hospitalarios se concentra en dos de las enfermedades que abordamos: diabetes e hipertensión (IMSS, 2014; ISSTE, 2016).

El simulador de salud consiste en estimar la probabilidad de que los individuos se enfermen de acuerdo con sus características individuales y antecedentes médicos, para con ello tener el número de personas que son y podrían ser diagnósticadas, así como aquellas que reciben o recibirían tratamiento. Al tener el total de personas a tratar, es posible calcular un gasto en salud, asignando a cada individuo un costo aproximado del tratamiento a 
recibir. Además, con esta herramienta se pueden plantear diversos escenarios en cuanto a la cobertura efectiva y el aumento o la disminución de costos.

El simulador que se contruye en este estudio se inserta en un simulador fiscal más completo, construido por el Centro de Investigación Económica y Presupuestaria (CIEP), el cual contiene la Ley de Ingresos de la Federación (LIF) y el Presupuesto de Egresos de la Federación (PEF), modelados para poder generar diferentes escenarios evaluativos del sistema fiscal y la sostenibilidad del mismo ${ }^{1}$.

Al modelar lo anterior, y en particular el simulador del sistema de salud, se hace posible entender la composición del gasto en la proporción correspondiente a estas tres enfermedades crónicas, y la probabilidad de que este aumente o disminuya basado en la probabilidad de que las personas contraigan diabetes, hipertensión o depresión y reciban el tratamiento correspondiente, así como por cambios en los factores de riesgo, tales como tomar, fumar u obesidad. El simulador es un primer esfuerzo que se hace en México, para la construcción de una herramienta que permite analizar el gasto en salud de diferentes esquemas de aseguramiento público, lo que genera información de distintos escenarios con el objetivo de construir un sistema universal de salud y su viabilidad financiera. Es decir, une información de atención a enfermedades determinadas y sus efectos en el presupuesto público.

Por tanto, este documento se organiza de la siguiente manera: en el siguiente apartado, se describe el sistema de salud en México; en el apartado dos, se hace una revisión de literatura sobre modelos de simulación en salud y esquemas de cobertura universal de salud en el mundo; el tercer segmento contiene la metodología que se sigue para construir el simulador de salud pública, basado en enfermedades crónicas; en el cuarto punto, se muestran los resultados de la construcción del simulador y un ejercicio aplicado de simulación; finalmente, en el apartado cinco, se emiten las conclusiones de este artículo y los pasos a seguir en la investigación.

\section{El sistema de salud en México}

El sistema de salud en México se compone de un sector público y un sector privado, en el que el sector público es financiado por los trabajadores, los empleadores y el Estado; mientras que el privado ofrece los servicios a la población con capacidad de pago (Gómez, Sesma, Becerril, Knaul, Arreola, \& Frenk, 2011). En este artículo, se abordará solamente el sector público, en

\footnotetext{
${ }^{1}$ CIEP.mx
} 
el que existen diferentes instituciones que proveen de atención médica: Instituto Mexicano del Seguro Social (IMSS), Instituto de Seguridad y Servicios Sociales de los Trabajadores del Estado (ISSSTE), Instituto de Seguridad Social para las Fuerzas Armadas (ISSFAM), Petróleos Mexicanos (PEMEX), Secretaría de Salud a través del Seguro Popular y las entidades proveedoras de salud en las entidades federativas.

El número de personas que están afiliadas a alguna institución de salud varía de acuerdo con la fuente de información. En 2015, los registros institucionales ${ }^{2}$ indicaban que había más personas afiliadas a un servicio médico que el total de la población del país. Esto podría sugerir que existe cobertura universal en salud y que todos los mexicanos cuentan con atención médica, la mayoría, por parte de las instituciones públicas (solo 1.8\% por instituciones privadas). Sin embargo, existen duplicidades en la condición de aseguramiento y una brecha entre el aseguramiento y la cobertura efectiva, la cual se refiere a la necesidad de atención de las enfermedades y la utilización por parte de los individuos de estos servicios, en tiempo y con la calidad requerida (CIEP, 2013).

Los resultados de la Encuesta Nacional de Salud y Nutrición de 2012 (INSP, 2012) señalan que aproximadamente 90.2 millones de personas reciben servicios de salud por parte de instituciones públicas y privadas. El Informe General de la ENSANUT 2012 especifica que existe $21.39 \%$ de la población sin atención médica.

El análisis que la Secretaría de la Función Pública lleva a cabo con los padrones de beneficiarios y derechohabientes (SFP, 2015), arroja coberturas parecidas a las auto-reportadas en la ENSANUT 2012, con un total de 97.09 millones de personas afiliadas a las instituciones de seguridad social y al Seguro Popular ${ }^{3}$.

Para reforzar el hecho de que todavía no hay cobertura total en salud, el Consejo Nacional para la Evaluación de la Política Social (CONEVAL) reporta que el porcentaje de personas sin acceso a servicios salud se ha reducido de $29.2 \%$ en 2010 a $18.2 \%$ en 2014 (CONEVAL, 2017).

\footnotetext{
${ }^{2}$ Los registros institucionales se calculan a través de estimaciones con base en coeficientes familiares, los cuales se interpretan como un promedio del número de miembros por familia y se aplican al número de asegurados directos y de pensionados (IMSS, 2014) .

${ }^{3}$ Los números reportados por la Secretaría de la Función Pública se refieren al número de derechohabientes y/o beneficiarios que las dependencias y entidades integran al Sistema Integral de Información de Padrones de Programas Gubernamentales (SIIPP-G).
} 
Respecto al gasto en salud, la institución con más presupuesto, año con año, es el IMSS, seguido del Seguro Popular. De 2012 a 2014, el presupuesto asignado a la función salud del Presupuesto de Egresos había crecido en promedio $4.5 \%$ en términos reales con respecto al presupuesto del año inmediato anterior. En 2015, se observó un incremento de $1.8 \%$ solamente, y para 2016, mostró una caída de $1.2 \%$, movimientos que indican que la política de cobertura universal en salud no ha sido prioritaria en los últimos años.

En 2015, las cifras de gasto per cápita por institución diferían drásticamente. El gasto promedio por asegurado en Seguro Popular es $20 \%$ menor que en IMSS, mientras que respecto del ISSSTE, la diferencia es 35\% menos. Lo anterior, es una señal, entre muchas otras, de la calidad, tipo y nivel diferenciado de atención que cada institución provee a sus afiliados (CIEP, 2013).

En cuanto a los factores que tienen un impacto directo en el gasto en salud, se encuentra la transición demográfica, la cual en todos los países del mundo tiene varios efectos en el ingreso y gasto de los gobierno. En este caso en particular, el cambio en la población de acuerdo con su edad se relaciona estrechamente con los servicios de salud que demandan actualmente, y demandarán en el futuro, debido a que las enfermedades se presentan en diferentes etapas de la vida y dependen también de los estilos de vida y hábitos de los individuos (factores de riesgo). En Deaton (2013), se menciona que anteriormente las enfermedades infecciosas eran las que atacaban principalmente a los niños, provocando que la esperanza de vida de la población en general fuera baja. La investigación e innovaciones en medicina han permitido que las tasas de mortalidad infantil disminuyan aun en países pobres, debido a que los descubrimientos en medicina son compartidos con todo el mundo. Así, las ganancias en esperanza de vida se han dado por esta caída en la mortalidad infantil, pero también por la atención a las enfermedades crónicas en adultos mayores, habitantes principalmente de países ricos. Efectivamente, se quiere vivir más y con mejor calidad de vida, por ello, no debe olvidarse la morbilidad, la cual se refiere a las enfermedades que padecerán la mayoría de aquellos niños que libraron la mortalidad infantil. Por tanto, el número de adultos que demandarán tratamientos por enfermedades crónicas aumentará, así como la necesidad de servicios de salud, acompañado por un mayor gasto público y privado, en este rubro (Deaton, 2013). 
Las proyecciones de población del Consejo Nacional de Población (CONAPO, 2015) ${ }^{4}$ indican que los 121 millones de mexicanos residiendo en el país en 2015, aumentarán a 134 millones en 2030, población que estará conformada por menos jóvenes y más personas en edad madura y vejez.

Como ya se mencionó, otro factor importante que afecta el gasto en salud es la transición epidemiológica, es decir, las enfermedades que se presentan en mayor medida entre la población, y su evolución a lo largo del tiempo. Actualmente, se observan bajas tasas de mortalidad, aumento en la esperanza de vida, caída en tasas de fecundidad y aumento de las enfermedades crónicas (INSP, 2012). En México, conforme a lo que establece el Institute for Health Metrics and Evaluation (IHME), las enfermedades o causas de muerte que predominaban en 1990 eran enfermedades del corazón, infecciones respiratorias, enfermedades diarreicas, diabetes, infarto y cirrosis. En 2010, desaparecen las enfermedades relacionada con diarrea y con infecciones respiratorias dentro de las principales causas de muerte, y aparecen enfermedades del riñón y cardiacas (IHME, 2013). Además, los informes financieros presentados en junio de 2015, tanto del IMSS como del ISSSTE, muestran que las enfermedades que presentan los derechohabientes con mayor frecuencia son enfermedades crónicas no transmisibles, como diabetes, hipertensión, enfermedades de los riñones y cáncer, entre otras.

De lo anterior, se infiere que el gasto en salud tenderá a aumentar debido a que la población, tanto por cambios demográficos, como por transición epidemiológica, presentará en mayor medida enfermedades crónicas cuyo tratamiento resulta costoso. Entre otros costos que la política de salud contempla y deberá seguir contemplando, están la salud preventiva y la atención materno-infantil. Tanto la salud como el ingreso son dos componente esenciales y complementarios del bienestar de las personas, el problema es que la elección de gastar en salud no es directa. Generalmente, las personas no están conscientes de lo que gastan en salud porque piensan que los empleadores pagan por ella, pero si las empresas tienen menos ganancias, los salarios disminuirán, afectando su nivel de bienestar. Además, los salarios han crecido de manera más lenta de lo que han crecido los costos en salud. Es un hecho que los servicios médicos de calidad permiten mantener y mejorar la salud, pero también son caros, lo que supone un cambio en prioridades relacionadas con el bienestar; es decir, para tener más salud es probable que se deje de viajar, por ejemplo. Sin embargo, Angus Deaton ha expuesto que el gasto en salud en diferentes áreas de Estados Unidos es variable y que la relación entre gasto y resultados de calidad puede ser negativa, probablemente porque hay médicos que piden más estudios y recetan más medicamentos sin que los resultados sean muy diferentes a

\footnotetext{
${ }^{4}$ Consultar: www.conapo.gob.mx
} 
cuando se gasta menos. Dado lo anterior, podría pensarse que el gasto en salud se reduciría sin afectar demasiado la salud de la población (Deaton, 2013).

\section{Revisión de literatura}

Dado que el objetivo de este artículo es construir una herramienta de microsimulación que permita evaluar la sostenibilidad del gasto en salud bajo escenarios como la cobertura universal, se hace una revisión de la literatura relacionada con lo que significa la cobertura universal en salud (efectiva o no), seguida del trabajo que se ha realizado en torno a los modelos de microsimulación.

\subsection{Cobertura universal}

La cobertura universal en salud o atención universal es una política pública que la Organización Mundial de la Salud (OMS) y el Banco Mundial (BM) han empujado para que los países alrededor del mundo la adopten, por estar estrechamente ligada al bienestar de la población. Este concepto está establecido en la Constitución de la OMS de 1948, donde se declara que la salud es un derecho humano fundamental, así como en el artículo 4to. de la Constitución Mexicana. De acuerdo con la Organización Mundial de la Salud, la cobertura universal en salud es asegurar que las personas reciban los servicios de salud que necesitan, sin sufrir gastos catastróficos al pagar por ella (OMS, 2014).

Para que un país se jacte de haber alcanzado la cobertura universal en salud, debería cumplir con lo siguiente: (i) contar con un sistema de salud sólido, eficiente, equitativo y bien administrado que identifique y trate las necesidades de salud a través de una atención integral centrada en la persona; esto es, informar y alentar a los individuos a permanecer saludables y prevenir enfermedades, detección temprana, tener la capacidad de otorgar el tratamiento pertinente y ayudar a los pacientes en la etapa de rehabilitación.

(ii) Dicho sistema debe ser financieramente sostenible, para que las personas no incurran en gastos que afecten su bienestar; lo que se puede lograr a través de diferentes esquemas que combinen proveedores de servicios de salud públicos y privados, por ejemplo. (iii) Asimismo, debe proveer de las medicinas y tecnología pertinentes para el diagnóstico y tratamiento de las enfermedades. (iv) Finalmente, debe contar con personal de salud capacitado y motivado para atender las necesidades de los pacientes (OMS, 2014).

Más que hablar solamente de cobertura, la OMS hace referencia a la cobertura efectiva y universal en salud, esto quiere decir: el beneficio real en 
salud que las personas reciben por medio de las intervenciones que el sistema de salud les provee, a partir de sus necesidades específicas.

El gasto público en salud depende directamente de la cobertura de las instituciones públicas. En México, se tiene la afiliación o aseguramiento de los individuos a distintos servicios de salud pública, como son IMSS, ISSSTE, ISSFAM, PEMEX y la Secretaría de Salud a través del Seguro Popular. Actualmente, alrededor de $75 \%$ de la población se encuentra asociado a alguna de estas instituciones (Urquieta-Salomón y Villarreal-Páez, 2015). Sin embargo, es más importante considerar la cobertura efectiva, la cual incluye la necesidad de la población por los servicios de salud y la utilización de los mismos. Por ejemplo, en Urquieta-Salomón et al. (2015), se presenta evidencia sobre la brecha que existe entre la cobertura efectiva y la afiliación, principalmente en la atención a los niños, lo que representa un reto importante para poder hablar de cobertura universal.

La cobertura efectiva universal es uno de los objetivos que plantean los organismos internacionales ${ }^{5}$, argumentando que tiene un impacto directo en la salud y bienestar de la población que les permite ser más activos y productivos en sus familias y comunidades. También asegura que los niños asistan a la escuela y los adultos trabajen para ganar un ingreso. Al mismo tiempo, busca ser una protección ante el riesgo financiero de gastar grandes cantidades en atención médica, empujando así a los más pobres a mantenerse en esa situación. La cobertura efectiva universal en salud debería ser una de las principales actividades de un gobierno comprometido a mejorar el bienestar de sus habitantes (OMS, 2014).

Sin embargo, lograr la cobertura efectiva universal, bajo las características descritas, resulta una tarea complicada por varias razones. En algunos países, el sistema de salud se desarrolló para los trabajadores formales, de manera fragmentada, interviniendo distintas instituciones de salud en la provisión de los servicios de salud con numerosos paquetes de salud. A veces, estas instituciones solamente proveen de manera gratuita la atención primaria, pero no la hospitalaria, la cual es más costosa y genera otros problemas para familias que no tienen la capacidad de pago. En otros países, se desarrollaron esquemas para la población pobre, pero no para el resto, lo que genera desigualdades e inequidad en varios sentidos. Pero, una de las razones más difíciles es encontrar la mejor forma para financiar un sistema de cobertura efectiva universal en salud, asegurando el acceso, la calidad, la equidad y la eficiencia de los servicios.

\footnotetext{
${ }^{5}$ Banco Mundial, 2015; World Bank, 2015; OMS, 2014.
} 
Alrededor del mundo, se han llevado a cabo esfuerzos para contar con un sistema de salud universal, como por ejemplo en Gana, Filipinas, Perú, Senegal, entre otros, los cuales han implementado diversas modalidades que no incorporan elementos de los modelos típicos de seguridad social. En general, sí es posible hablar de dos modelos de seguridad social que incluyen la provisión de servicios de salud: Bismark y Beveridge. El primero fue adoptado por Alemania en 1883; el segundo se implanta en el Reino Unido, en plena guerra mundial (1942). El modelo Bismark no pretende proveer servicios médicos a toda la población, mientras que el modelo Beveridge sí contempla tal objetivo. La manera de financiarse es diferente, el primero se hace a través de cuotas y pagos complementarios para servicios de hospitalización y farmacéutico, mientras que el segundo lo hace a través de impuestos pagados por toda la población. Otra diferencia es que en el modelo Beveridge, los sistemas de salud están integrados, lo que hace que la provisión de servicios sea uniforme; además, el personal médico lo conforman empleados públicos (PiPP, 2011).

En el caso de los países mencionados, las dimensiones que se utilizan para medir los avances en la cobertura universal son, generalmente: (i) la expansión en la cobertura, es decir, a quiénes cubre el sistema de salud; (ii) la expansión de la oferta de servicios de salud a la población o, en otras palabras, qué tipo de enfermedades y tratamientos se cubren, y (iii) la ampliación de la cobertura financiera, qué proporción de los costos estará cubierta por el estado y qué proporción por los ciudadanos. En ocasiones, se añade un componente sobre economía política que involucra el diseño institucional de cada país y su práctica organizacional, así como, otro componente sobre la igualdad y equidad de la provisión de los servicios (Lagomarsino, Garabrant, Adyas, Muga, y Otoo, 2012).

Dentro de las acciones que han llevado a cabo distintos países para aumentar la cobertura universal en salud, se encuentra el aumento del presupuesto en salud entre $5 \%$ y $11 \%$ en países como Gana, Indonesia, Ruanda y Vietnam, a pesar de contar con altas proporciones de personas que laboran en el sector informal, lo que hace más difícil cobrar impuestos y cuotas de seguridad social. Algunos países, sobre todo aquellos que destinan subsidios a poblaciones específicas (Gana, India y Filipinas), acostumbran financiar la expansión de su sistema de salud, a través de impuestos generales; mientras que otros (Kenia, Nigeria, Gana), tratan de recolectar aportaciones voluntarias de parte de los hogares. También, muchos países (Gana, Filipinas, Indonesia) se han decidido por un solo programa de salud para toda la población, en vez de tener programas o paquetes separados para distintas sub poblaciones. Asimismo, aquellos países que solo proveían servicios 
hospitalarios, ampliaron su cobertura a atención preventiva y primaria, porque consideran que estos son menos $\operatorname{costosos}^{6}$. En este mismo sentido, crearon agencias independientes para comprar los servicios de salud a proveedores públicos y privados.

En cuanto a la cobertura o a quiénes cubren los sistemas de salud en los países que se han hecho cambios, se encuentra que ampliaron la cobertura llegando a $76 \%$ en Filipinas, $63 \%$ en Indonesia y $60 \%$ en Perú, pero las desigualdades persisten tanto en la calidad de los servicios como en la provisión de los mismos a distintas poblaciones (pobres, adultos mayores, niños) (Soors, Devadasan, Durairaj, y Criel, 2010). En relación con la proporción de costos de los servicios que pagan los hogares cuando realizan las acciones dirigidas a la cobertura universal, se tiene que, en la mayoría de los países que se encuentran en una etapa intermedia de reformas (más de 10 años), el gasto de bolsillo de los hogares decrece entre 3 y 6 puntos porcentuales, pero este gasto se encuentra todavía alto con respecto a lo recomendado por la OMS (15-20\% del total de gasto en salud) (Lagomarsino et al., 2012).

Con el fin de tener una cobertura universal, o mejor dicho, una afiliación universal, en México, se creó en 2004 el Sistema de Protección Social en Salud (SPSS), el cual cuenta con un componente financiero principal: el Seguro Popular (SP). Este seguro público es voluntario, no depende del estatus de salud preexistente y es gratuito para los hogares ubicados en los primeros deciles de ingresos (hasta el sexto decil). Uno de los principales objetivos del SP ha sido proteger a los hogares que no cuentan con algún tipo de cobertura, ante la necesidad de incurrir en gastos de bolsillo, de modo que no haya obstáculos económicos para acceder a los servicios de salud (ÁvilaBurgos, Serván-Mori, Wirtz, Sosa-Rubí, \& Salinas-Rodríguez, 2013). La cobertura de la población, medida como afiliación a algún seguro médico, creció de alrededor de 60\%, antes de la creación del SP, a 97\% en 2015. Sin embargo, aunque la cobertura aumentó, la población padece de la misma desigualdad e inequidad en lo que a la provisión de servicios de salud respecta, tanto en calidad como en cantidad, debido a que los paquetes de salud que se proveen en el SP son diferentes a los que se proveen en las otras instituciones de salud.

\footnotetext{
${ }^{6}$ La evidencia de que la atención primaria y preventiva es más barata no es definitiva (Musgrove, 1987; Bray, Jemal, Torre, Forman, \& Vineis, 2015; Guzmán \& VargasHernández, 2013), sin embargo, la práctica mundial es seguir invirtiendo en prevención y atención primaria, en parte debido a los compromisos adquiridos en Alma Ata.
} 
En México, el gasto de bolsillo, uno de los fenómenos que se quieren abatir con la cobertura universal y, en este caso, uno de los principales objetivos del SP, se ubicaba en 58\% del gasto total en 2003 (Ávila-Burgos et al., 2013), para 2015, esta proporción fue 45\% (OECD, 2015). Aunque el gasto de bolsillo ha disminuido, sigue siendo el más alto de los países de la Organización para la Cooperación y el Desarrollo Económicos (OCDE).

\subsection{Modelos de microsimulación}

Una de las vías para modelar el gasto en salud pública es a través de modelos de microsimulación de salud. Los modelos de microsimulación permiten simular los efectos de una política en una muestra de agentes, como individuos, hogares, empresas, entre otros. Los modelos evalúan las consecuencias de un cambio en el entorno económico, producido por un cambio en la política, en un vector de indicadores de actividad o bienestar de los individuos (Bourgignon y Spadaro, 2006). En el caso de salud, se trata de simular eventos o historias individuales asociados con componentes clave de un proceso de enfermedad. Estas historias simuladas pueden analizarse de manera agregada para estimar los efectos de un tratamiento en cierta enfermedad, en nivel de población, y también comparar la efectividad de distintos tratamientos (Rutter, Zaslavsky, y Feuer, 2011). La microsimulación se ha usado con mayor frecuencia para proveer de información que permite dirigir la política pública.

Se han encontrado diversos estudios que utilizan modelos de microsimulación para estimar la incidencia de diferentes enfermedades, así como el cambio e impacto de los factores de riesgo en dicha incidencia de acuerdo con el cambio demográfico en distintos países (Manuel, y otros, 2014) (Lymer \& Brown, 2012) (Pichon-Riviere, Augustovski, Bardach, Colantonio, \& Group3, 2011). Sin embargo, muy pocos estudios incorporan en el modelo de microsimulación los costos de tratamiento de distintas enfermedades, dejando a un lado la posibilidad de observar qué sucede con el gasto y el financiamiento de los servicios de salud.

Asimismo, existen modelos de microsimulación que se utilizan para estimar la relación costo-efectividad de algunas intervenciones en el tratamiento de enfermedades crónicas, desarrollados principalmente en Canadá, Estados Unidos y Europa. El Population Health Model (POHEM) es un modelo de microsimulación liderado por Statistics Canada, el cual proyecta el uso de servicios de salud para atender ciertas enfermedades crónicas como cáncer de pulmón, diabetes o enfermedades cardiovasculares. Este modelo no proyecta el gasto en salud total, sino que provee de una herramienta para evaluar, a través de distintos escenarios, los impactos en salud y en el sistema de salud de nuevos tratamientos, a cambios en política pública dirigidos a abatir 
ciertos factores de riesgo o incidencia en enfermedades. POHEM construye una población canadiense virtual representativa basada en individuos quienes experimentan eventos de salud como el desarrollo de una enfermedad o factores de riesgo, recepción de tratamientos o envejecimiento. Los factores de riesgo se modelan de manera explícita (cambios en comportamiento de fumadores, obesidad, hipertensión, etc). Es un modelo de microsimulación dinámica en tiempo continúo, basado en un proceso de Montecarlo en donde la ocurrencia de eventos es aleatoria y los efectos en los individuos presentan diferencias. De las variables incorporadas al modelo para calcular el gasto en salud, se encuentran la dinámica poblacional, la distribución de los factores de riesgo, la incidencia e intensidad de las enfermedades, el uso de diagnósticos y terapias, y el costo de tratamientos. Finalmente, las fuentes de información utilizadas para el POHEM son encuestas de salud representativas en el nivel nacional, bases de datos de hospitalización, censos y bases de datos de costos de salud, así como parámetros determinados en la literatura (Statistics Canada, 2015).

Otro modelo es aquel desarrollado por la Universidad de Carolina del Sur y RAND, entre otros, en Estados Unidos, cuyo nombre es Future Elderly Model (FEM). FEM proyecta el total de gastos (Medicare, Medicaid, medicinas, etc) de la población de 51 años y más considerando el cambio demográfico, el estatus de salud y las tendencias en discapacidad. Entonces, simula y evalúa posibles cambios en las tendencias de salud debido a cambios en el sistema de salud, en el estatus de salud de los adultos mayores, innovaciones médicas, disminución en las enfermedades crónicas o en la cantidad de adultos con obesidad. La población del modelo se obtiene de la encuesta de beneficiarios de Medicare y cuenta con tres sub-modelos principales: (i) Modelo para las transiciones de estatus de salud (mortalidad, cáncer, enfermedades cardiovasculares, diabetes, entre otras). (ii) Modelo que predice las características de salud que cambian año con año, asegurando que la población de 51 años o más permanece representativa; si se usa la prevalencia de enfermedades por edad y la trayectoria de enfermedades es posible predecir el estatus de salud de las siguientes generaciones. (iii) Modelo de mínimos cuadrados ordinarios para costos de salud que incorpora información sobre factores de riesgo y la presencia de enfermedades crónicas; asimismo, las variables que influyen en el gasto de salud, son: la dinámica poblacional, la distribución de los factores de riesgo, las enfermedades crónicas y el costo anual de los tratamientos (USC, 2011).

En el Reino Unido, se desarrolló el modelo National Heart Forum Microsimulation Model (NHFM) para proyectar el gasto en salud de las principales enfermedades derivadas de la obesidad simulando el cambio en las tendencias de la prevalencia de estas enfermedades, debido a políticas dirigidas a reducir la obesidad en la población. El modelo se construye sobre 
una población inglesa virtual y representativa, que nace en la simulación, experimenta eventos de salud como el desarrollo de factores de riesgo y enfermedades y muere. El modelo se desarrolla en tres pasos: (i) análisis de regresión para estimar la distribución de los factores de riesgo en la población futura; (ii) modelo de microsimulación que proyecta la carga de enfermedades asociadas a los factores de riego estimados y (iii) cálculo posterior para obtener los gastos asociados a la carga de enfermedades basado en el costo promedio por paciente por enfermedad del Sistema Nacional de Salud Inglés (Wang, McPherson, Marsh, Gortmaker, y Brown, 2011). De igual manera que los modelos anteriores, las variables clave que se relacionan con el gasto en salud, son: la dinámica poblacional, el índice de masa corporal, la prevalencia de enfermedades relacionadas con obesidad y el costo anual de cada enfermedad; datos tomados de la literatura y de los datos nacionales.

El gasto social en América Latina ha crecido significativamente de 2005 a 2010 de \$461 dólares per cápita a \$1,026 (122\%). De 11.2\% a 18.6\% del PIB (salud, educación y protección social) (Glassman y Zoloa, 2014). Este crecimiento se ha dado por diferentes razones, las cuales se pueden clasificar en aquellas que son inerciales y aquellas que se relacionan con el diseño de las políticas públicas. Las primeras son estructurales, como el envejecimiento de la población, urbanización, disponibilidad de tecnología para desarrollar medicina avanzada y el acceso a nuevas drogas. Las segundas están relacionadas con la política pública, como decidir las intervenciones, poblaciones objetivo y subsidios. Para poder estimar cuánto cuesta proveer de servicios de salud a la población ahora y en el futuro, Glassman y Zoloa (2014) desarrollaron un modelo de microsimulación que incorpora información sobre tendencias en la población, factores de riesgo, características socioeconómicas y crecimiento tecnológico en Brasil, Chile y México. Estos autores definen el gasto en salud como la suma de los gastos relacionados con la salud de los individuos de una población, tomando en cuenta la probabilidad de que un individuo se enferme y sea tratado por esa enfermedad.

\section{Metodología}

Este estudio propone modelar una herramienta de microsimulación que incorpore un análisis tanto de perfiles epidemiológicos y demográficos de la población, como el costo de las enfermedades y el gasto público en salud relacionado. Para ello, se seguirá la metodología planteada en (Glassman y Zoloa, 2014). Se define el gasto en salud, de la siguiente manera:

$$
\text { Gasto }_{t}^{e}=\sum_{f}^{F} \sum_{i}^{N} P_{i}^{f}\left(x_{i}^{e} \beta_{t 0}^{f}\right) * P_{i}^{T f}\left(T_{i}^{e} \delta_{t 0}^{f}\right) * C E_{t 0}^{f}
$$


donde $\mathrm{P}_{\mathrm{i}}^{\mathrm{f}}\left(\mathrm{x}_{\mathrm{i}}^{\mathrm{e}} \beta_{\mathrm{t} 0}^{\mathrm{f}}\right)$ es la probabilidad de que le individuo $\mathrm{i}$ contraiga la enfermedad $\mathrm{f}$, la cual depende de las características del individuo y los coeficientes obtenidos a través de un modelo probit en t0; $\mathrm{P}_{i}^{\mathrm{Tf}}\left(\mathrm{T}_{\mathrm{i}}^{\mathrm{e}} \beta_{\mathrm{t0}}^{\mathrm{f}}\right)$ es la probabilidad de que un individuo i con la enfermedad $f$ sea tratado; $\mathrm{CE}_{\mathrm{t} 0}^{\mathrm{f}}$ se refiere al costo promedio del tratamiento en t0.

Por tanto, el gasto en salud es la sumatoria del costo de las enfermedades multiplicado por la probabilidad de que un individuo se enferme y sea tratado en un escenario específico (e) y tiempo específico (t).

Las probabilidad de enfermarse de cierta enfermedad y de ser tratado se estimarán por edades y sexo, así será posible obtener estas mismas probabilidades para los años subsecuentes con base en las proyecciones demográficas de CONAPO.

Las probabilidades de enfermedad y tratamiento se estimarán a través de modelos de regresión probit, de los cuales es posible obtener los coeficientes que explican la ocurrencia de un evento, a partir de características del individuo y del entorno.

$$
\begin{aligned}
\text { Enfermarse }_{i, f} & =\alpha_{i, f}+\beta_{i, f} x_{i}+\varepsilon \\
\text { Tratamiento }_{i, T f} & =\gamma_{i, T f}+\delta_{i, T f} T_{i}+\varepsilon
\end{aligned}
$$

Por tanto, contraer o no la enfermedad $\mathrm{f}$ dependerá de distintas características del individuo y el entorno $\left(\mathrm{x}_{\mathrm{i}}\right)$, como: edad, sexo, estado civil, escolaridad, si el individuo es indígena, si trabaja, antecedentes de la enfermedad en padre y madre, si radica en zonas rurales, factores de riesgo (fuma, toma o padece obesidad).

Asimismo, recibir tratamiento para curar la enfermedad $f$ depende de características del individuo y del tratamiento $\left(\mathrm{T}_{\mathrm{i}}\right)$, tales como edad, escolaridad, institución proveedora de servicios médicos (afiliación) e ingreso, condicionado a haber sido diagnosticado con la enfermedad.

Adicionalmente, se estimaron ecuaciones para obtener la distribución probabilidad de que una persona fume, tome o padezca de obesidad, igualmente, por medio de modelos probit. En estos modelos, las variables independientes, son: edad, sexo, estado civil, escolaridad, si el individuo es indígena, si trabaja, si radica en zonas rurales y si fuma o toma. Estos modelos se incorporan para poder hacer simulaciones con escenarios donde la proporción de individuos que fuma o toma, aumenta o disminuye por la intervención de alguna política pública. 
Cabe mencionar que los modelos se corrieron por sexo, es decir, se calcula la probabilidad de enfermarse y de ser tratados para cada una de las enfermedades, para mujeres y hombres por separado.

\section{Datos}

La Encuesta Nacional de Salud y Nutrición (INSP, 2012) se levantó en el año 2012 con el propósito de cuantificar la frecuencia, distribución y tendencias de las condiciones de salud y nutrición, así como sus determinantes. También contiene información sobre la cobertura de protección en salud de los individuos, la utilización y condiciones de vida de acuerdo con los diferentes servicios de salud que existen en nuestro país. Esta encuesta se ha llevado a cabo en 2006 y 2012.

Para este trabajo en específico, se utiliza la ENSANUT 2012, porque contiene información sobre algunas de las enfermedades que la población presenta en mayor medida, como lo son: diabetes mellitus, depresión, hipertensión arterial, enfermedades cardiovasculares, hipercolesterolemia y enfermedades neurovasculares. Estas enfermedades coinciden con las 10 principales enfermedades que reporta atender el IMSS, en el Informe al Ejecutivo Federal y al Congreso de la Unión, sobre la situación financiera y los Riesgos del Instituto (IMSS, 2014). De igual forma, es posible contar con información relacionada con el tratamiento de las enfermedades mencionadas anteriormente: si recibió el tratamiento, qué tipo de tratamiento y qué institución se lo brindó. En algunos casos, se conoce la razón por la que el individuo no recibió el tratamiento. Por otro lado, la encuesta también contiene información sobre factores de riesgo como obesidad y si los individuos fuman o toman, entre otros factores. Por tanto, se utilizó el módulo de hogares, individuos y adultos, el cual contiene la información sobre el padecimiento de enfermedades crónicas y su tratamiento para 46,277 registros de individuos mayores de 20 años. La ENSANUT 2012 es una encuesta probabilística que permite ofrecer resultados representativos para cada una de las entidades del país, para los ámbitos urbano y rural, y para el país en su conjunto (INSP, 2012).

El cuadro 1 muestra que $9.1 \%$ de la población mayor a 20 años está diagnosticada como diabética y de ellos, $85.5 \%$ recibe tratamiento, independientemente de la institución proveedora. $15.9 \%$ de los adultos son hipertensos y solamente $67.7 \%$ es atendido para controlar esta enfermedad, mientras que del $10 \%$ de personas que sufren de depresión, $65.5 \%$ recibe tratamiento.

Sobre los factores de riesgo, aproximadamente $22.5 \%$ de la población mayor a 20 años presentan obesidad, $36.5 \%$ fuma y $41.7 \%$ toma alcohol. 
Cuadro 1

Proporción de enfermos, tratados y factores de riesgo. Statu Quo

$\begin{array}{ll}\text { Proporción de } & \text { Proporción de enfermos } \\ \text { Enfermos (\%) } & \text { con tratamiento }(\%)\end{array}$

\begin{tabular}{lcc}
\hline Diabetes & 9.17 & 85.53 \\
Hipertensión & 15.92 & 67.73 \\
Depresión & 10.72 & 65.54 \\
\hline \multicolumn{3}{c}{ Factores de riesgo } \\
\hline Obesidad & 22.48 \\
Fuma & 36.45 \\
Toma & 41.75 \\
\hline
\end{tabular}

Fuente: Elaboración propia con datos de la ENSANUT 2012 (INSP, 2012)

Otra posible fuente de información sería la Encuesta de Ingreso y Gasto de los Hogares (ENIGH), la cual también levanta información sobre salud. Sin embargo, esta se refiere solamente a la afiliación y algunas preguntas sobre control de peso, y detección de diabetes o presión alta sin incluir si la enfermedad fue diagnosticada o si ha sido tratada por alguna de las instituciones que brindan servicios de salud pública en el país.

Una forma de estimar el costo o gasto público por individuo es tomando el gasto total asignado a cada una de la instituciones de salud y dividirlo entre el número de afiliados que cada una de ellas tiene. Sin embargo, esta medida no toma en cuenta el número de personas que necesitan servicios médicos, que se enferman y que se atienden. Por tanto, es necesario calcular el costo promedio por enfermedad, es decir, cuánto cuesta atender a una persona que padece diabetes, por ejemplo, en promedio. Para lograr lo anterior, se parte de los Grupos Relacionados con el Diagnóstico (GRD), ejercicio que clasifican casos por tipo de diagnóstico (primario y secundario), características del paciente (sexo, edad), presencia de morbilidad y complicaciones, y procedimientos aplicados. Los casos clasificados bajo el mismo GRD se caracterizan por ser homogéneos, por tanto, son económica y médicamente similares. Las dos características básicas de un sistema de pago basado en GRD son: a) un sistema de clasificación detallado del paciente y b) una fórmula de pago que se basa en el peso relativo de los distintos grupos (Paolillo et al., 2008). Los GRD utilizados para esta investigación fueron elaborados por el IMSS y se toman en cuenta el costo promedio de la diabetes y la hipertensión con y sin complicaciones, así como de la neurosis depresiva. Los costos unitarios por enfermedad resultantes de la metodología 
GRD incluyen los recursos utilizados en atención hospitalaria: medicamentos, material de curación, laboratorio de patología, gabinete, banco de sangre, bienes de inversión y consumibles médicos. Por tanto, los costos utilizados en este artículo no contemplan los costos de la atención ambulatoria (IMSS, 2012).

En definitiva, el tema sobre el costo de las enfermedades es un tema de investigación en sí mismo. En este trabajo, se toman los costos de GRD como referencia.

\section{Cuadro 2}

Costos promedio por año de tratamiento de enfermedades crónicas en 2015

Enfermedad

Costo promedio (pesos de 2015)

Diabetes (con y sin complicaciones) 45,300

Hipertensión (con y sin complicaciones) 45,700

Depresión

61,205

Fuente: IMSS (2008) en pesos de 2015

Existen estudios en los que se estipula que los costos de estas enfermedades se encuentran alrededor de los promedios que se muestran en el cuadro 2. Por ejemplo, en Villarreal-Ríos y otros (2011), se establece que el costo promedio de la hipertensión y la diabetes se ubicaba en US\$5,862 y US\$2, 548 dólares, respectivamente. Asimismo, en Bolaños, Shigematsu, Ruíz, Márquez y Ávila (2010), se señala que el costo promedio anual por paciente fue de US $\$ 3,193.75$, correspondiendo US $\$ 2,740.34$ para el paciente sin complicaciones y US $\$ 3,550.17$ para el paciente con complicaciones. Los días/cama en hospitalización y en unidad de cuidados intensivos fueron los servicios con mayor costo. Los costos en otros países parecen ser más altos, como lo muestra la American Diabetes Association, quien indica que el tratamiento de diabetes es alrededor de USD $\$ 5,900$.

Finalmente, el gasto público se obtiene del Presupuesto de Egresos de la Federación (PEF) 2015. El PEF contiene los recursos aprobados por poder legislativo para que el poder ejecutivo pueda operar las políticas públicas correspondientes, entre ellas, la provisión de servicios de salud.

Debido a que los datos son de distintos años, los costos se convierten a valores de 2015, para ser congruentes con los datos del PEF. En cuanto a la 
información de la ENSANUT, se asume que la proporción de personas mayores de 20 años, que son diagnosticadas con alguna de las enfermedades en estudio, se mantiene constante en los siguientes tres años ${ }^{7}$.

\section{Resultados}

Los resultados de las regresiones, para estimar la probabilidad de ser diagnosticado por una de las tres enfermedades crónicas incluidas en este análisis, se muestran en las gráficas 1 a 3 . Cada una de las gráficas presenta la relación que existe entre las variables independientes y la probabilidad de que un individuo tenga diabetes, hipertensión o depresión, para hombres y para mujeres de manera independiente. Las variables independientes incluidas en las regresiones son: edad, edad al cuadrado, si el individuo está casado, si es indígena, los años de escolaridad, si tiene un trabajo, si consume (toma) alcohol, si fuma, si habita en zonas rurales, si padece de obesidad, así como, los antecedentes médicos familiares para las enfermedades con tal información.

Asimismo, se presentan las estimaciones de la probabilidad de que las personas diagnosticadas con alguna de las enfermedades crónicas estudiadas reciban tratamiento (gráfica 4). En este caso, las regresiones incluyen información sobre edad, institución proveedora de servicios de salud o afiliación y el ingreso de los individuos.

Por otro lado, se estima la presencia de factores de riesgo como son tomar alcohol, fumar y la obesidad, la cual, aunque no se considera como una enfermedad, también es un factor de riesgo, ya que contribuye al desarrollo de enfermedades crónicas como las estudiadas en este documento (gráficas 5 y 6).

Todas las interacciones de los modelos estimados en esta sección son significativas al nivel de $95 \%$ de confianza. Los resultados de los probit se pueden consultar en el apéndice 1.

Las gráficas se interpretan de la siguiente manera: los puntos por arriba de la línea del cero indican que esa variable se relaciona positivamente con la probabilidad de tener diabetes (fumar, tomar, etc.). Por ejemplo, en la gráfica 1, la edad, tanto para hombres como para mujeres, incrementa la probabilidad de contraer diabetes. Por otro lado, los puntos por debajo de la línea de cero,

\footnotetext{
${ }^{7}$ De hecho en la ENSANUT 2016, la proporción de enfermos de diabetes e hipertensión no son significativamente diferentes a la proporción de enfermos de diabetes e hipertensión de la ENSANUT 2012 (OMENT, 2016).
} 
con signo negativo, indican que esa variable se relaciona negativamente con la probabilidad de tener diabetes (fumar, tomar, etc.). Por ejemplo, la escolaridad tiende a disminuir la probabilidad de presentar la enfermedad o factor de riesgo. Aunque en las gráficas existen datos sobre la línea del cero, que indicarían que el parámetro es igual a cero, éstos son muy pequeños, pero significativos.

En el caso de la diabetes, los factores que inciden positivamente en la probabilidad de que se desarrolle esta enfermedad son la edad, el ser indígena, el consumo de alcohol, la obesidad y el que los padres hayan sido diabéticos también, tanto para hombres como para mujeres. Aunque se debe resaltar que en los hombres, la edad tiene un efecto mayor que en las mujeres (1.3 veces), así como la educación, la cual disminuye 42 veces más la probabilidad de que las mujeres sean diagnosticadas con diabetes, comparado con los hombres (coeficiente para hombres 0.0008 ; coeficiente para mujeres 0.036). Es decir, es menos probable que las mujeres con más años de escolaridad tengan esta enfermedad. La obesidad también afecta en mayor medida a los hombres. El estar casado tiene un efecto diferenciado por sexo, es positivo para hombres, pero negativo para mujeres. De acuerdo con el modelo desarrollado, la probabilidad de que un hombre mayor de 20 años sea diagnosticado con diabetes es $9.09 \%$, la misma medición para mujeres es $10.4 \%$.

Para la hipertensión, la edad, estar casado, tomar, fumar, la obesidad y si los padres padecieron de presión alta son determinantes que aumentan la probabilidad de ser hipertenso. También se encuentran efectos diferenciados por sexo, en cuanto a ser indígena y los años de escolaridad. En este caso, la probabilidad de presentar hipertensión disminuye para las mujeres mejor educadas, pero para los hombres, esta característica aumenta la probabilidad. De igual manera, un hombre indígena tiene menos probabilidad de padecer hipertensión en comparación con una mujer indígena. Tener un trabajo decrece 2.35 veces más la probabilidad de padecer hipertensión en comparación con una mujer. En cuanto a los efectos de los factores de riesgo, estos son más severos para las mujeres que para los hombres. La probabilidad de sufrir hipertensión para un hombre mayor a 20 años es $13.6 \%$, pero para las mujeres es $19.8 \%$. 
Gráfica 1

Probabilidad de tener diabetes

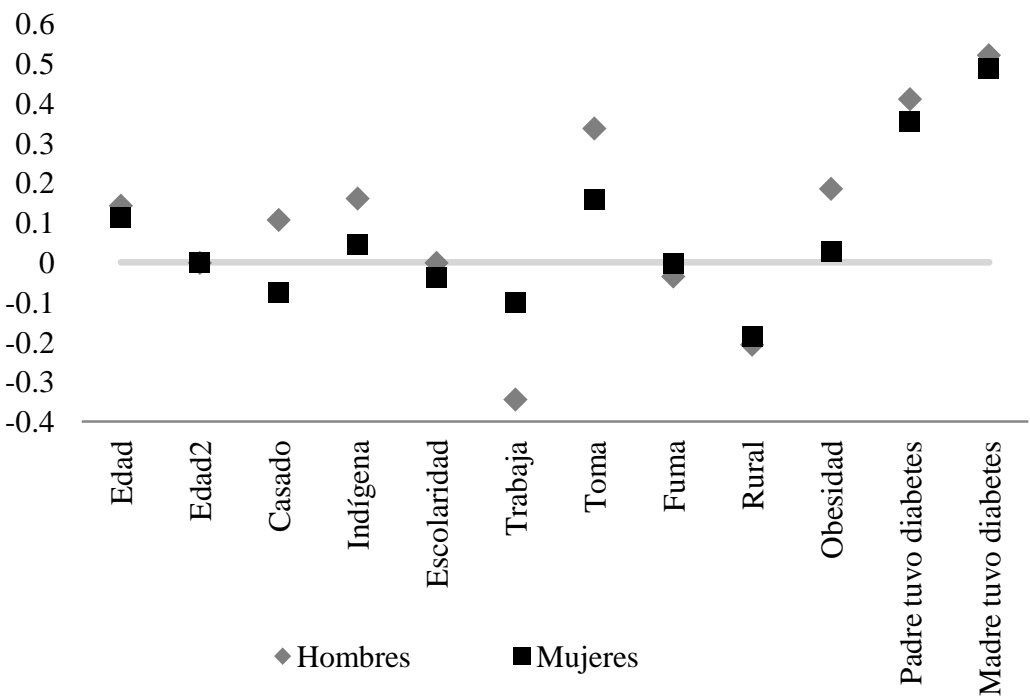

Fuente: Elaboración propia con datos de la ENSANUT 2012 (INSP, 2012)

Gráfica 2

Probabilidad de tener hipertensión

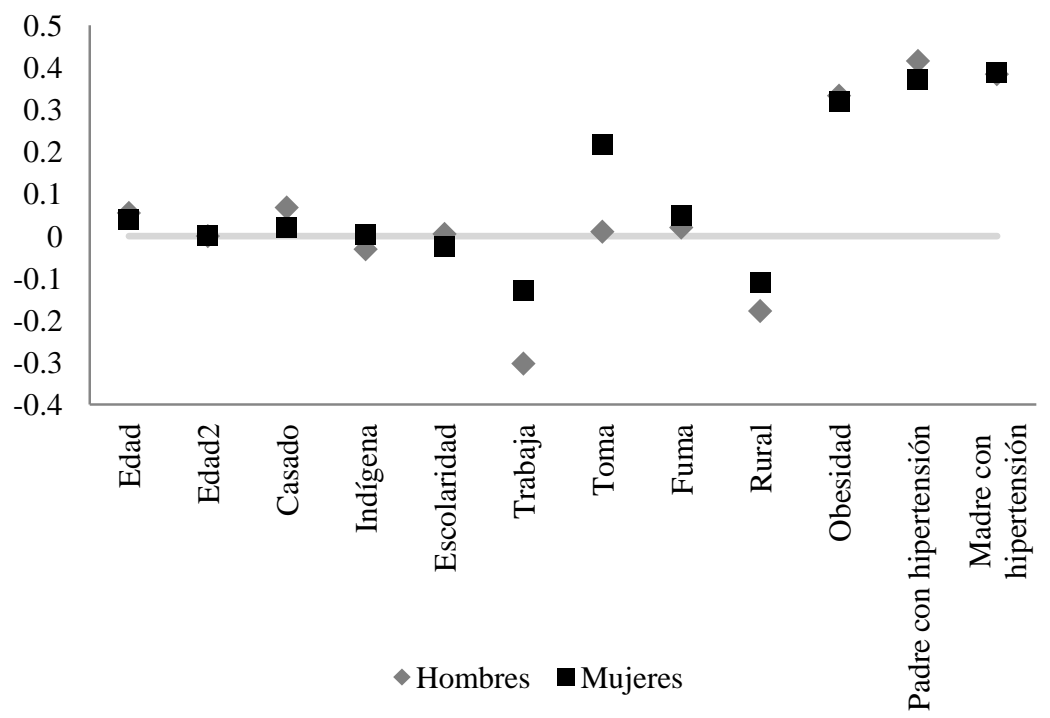

Fuente: Elaboración propia con datos de la ENSANUT 2012 (INSP, 2012) 
En cuanto a la depresión, enfermedad que se incluyó por su creciente incidencia y porque existe información relevante en la ENSANUT 2012. Las variables que tienen relación positiva con la probabilidad de tener depresión son la edad, los factores de riesgo (fumar y tomar) y la presencia de enfermedades crónicas. La obesidad, y el ser indígena tienen efectos diferentes para hombres que para mujeres. En el primer caso, es más probable que las mujeres desarrollen depresión, a que los hombres con obesidad la desarrollen. En el segundo caso, los hombres indígenas son más propensos a la depresión que las mujeres indígenas. A diferencia de la diabetes y la depresión, es más probable que las mujeres presenten depresión conforme se hacen mayores, comparado con los hombres. De igual manera, la escolaridad disminuye 13.7 veces más la probabilidad de deprimirse para mujeres que para hombres, mientras que el trabajo tiene efectos de mayor magnitud en la reducción de la probabilidad para hombres. Esta medida se ubica en 5\% para hombres y $14 \%$ para mujeres.

Para la probabilidad de ser diagnosticado con diabetes, hipertensión y depresión, tener un trabajo y vivir en zonas rurales se relacionan negativamente, es decir, disminuyen estas probabilidades para hombres y mujeres.

La estimación de las probabilidades de recibir tratamiento si una persona es diagnosticada como diabética o hipertensa se presenta en la gráfica 4. En estos modelos, se incluyen como variables independientes la edad, la edad al cuadrado, la escolaridad, la institución proveedora de servicios médicos a la que el individuo está afiliado y el ingreso del hogar. En general, se observa que es más probable que las mujeres reciban tratamiento para diabetes que los hombres, a través de las instituciones de salud, mientras que el tratamiento de hipertensión es más probable que lo reciban los hombres. La afiliación a PEMEX o ISSSFAM aumenta más de 2 veces la probabilidad de recibir tratamiento para diabetes, así como para recibir tratamiento para la hipertensión, para ambos sexos, comparado con la atención de estas enfermedades en el IMSS o ISSTE. La probabilidad de recibir tratamiento se reduce si están afiliados a IMSS-oportunidades, en especial para hombres diabéticos y para mujeres con presión alta.

La probabilidad de recibir tratamiento para ambas enfermedades aumenta con la edad de los individuos y con el ingreso de los hogares, es decir, entre mayor es el ingreso es más probable que las personas que padecen diabetes o hipertensión busquen atención médica.

Cabe mencionar que este análisis puede presentar problemas de endogeneidad, dado que el diagnóstico y tratamiento de la diabetes depende de la disponibilidad y asistencia de las personas a acudir a una de las 
instituciones de salud. Además, la probabilidad de tratamiento aumenta en instituciones que proveen servicios de salud a trabajadores del sector formal.
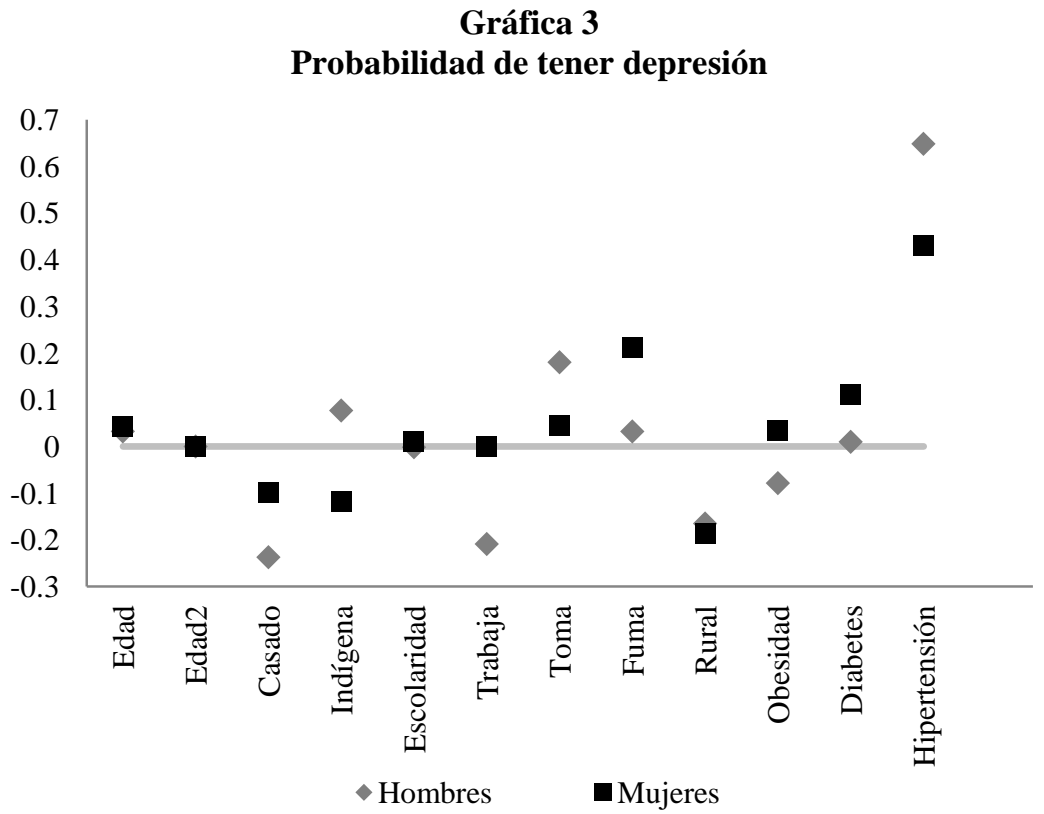

Fuente: Elaboración propia con datos de la ENSANUT 2012 (INSP, 2012)

Con respecto a los factores de riesgo: consumo de alcohol y tabaco, los resultados se presentan en la gráfica 5. Lo que se puede concluir es que fumar tiene un efecto positivo en la probabilidad de consumir alcohol y viceversa, mientras que tener trabajo también aumenta la probabilidad de tener estos comportamientos que se definen como factores de riesgo. Estas ecuaciones se calculan para poder distribuir la probabilidad de ocurrencia de los factores de riesgo en la población mayor de 20 años, para que, con ello, sea posible hacer cambios que generen diferentes escenarios a evaluar.

Finalmente, y de la misma manera que la estimación de la probabilidad de presentar alguna enfermedad, se calcula la probabilidad de presentar obesidad como factor de riesgo. Por tanto, la probabilidad de tener un índice de masa corporal alto aumenta con la edad, con el consumo de alcohol y tabaco, así como con la presencia de otras enfermedades crónicas. Cabe mencionar que tener un trabajo disminuye la probabilidad de presentar obesidad. 
Gráfica 4

Probabilidad de recibir tratamiento para diabetes e hipertensión.

a) Diabetes

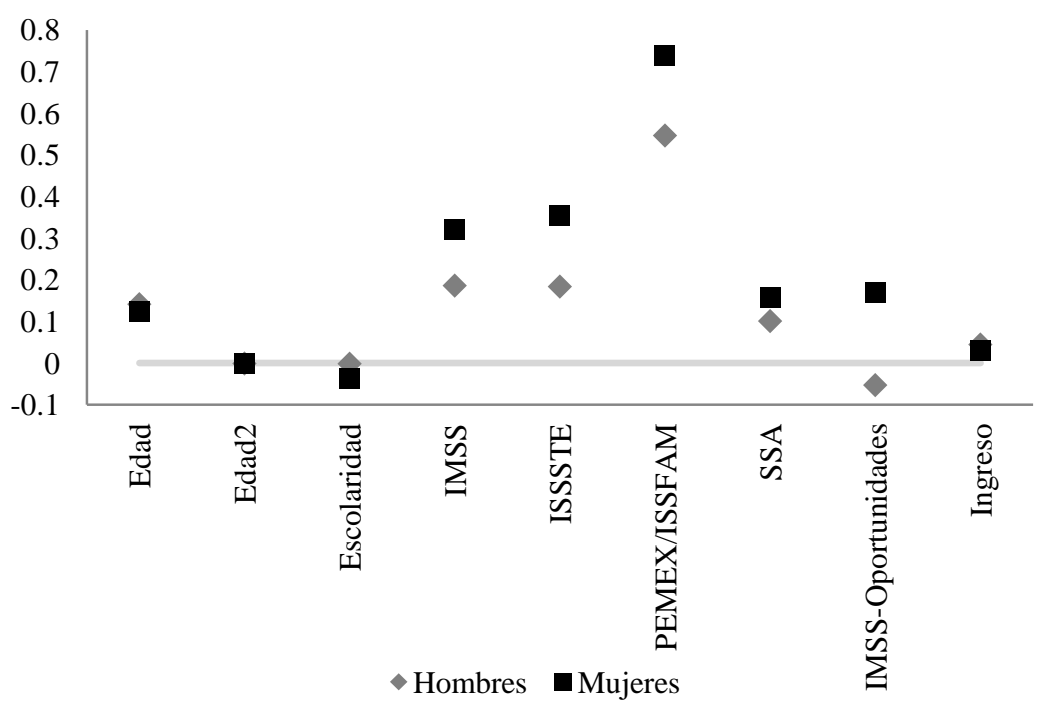

b) Hipertensión

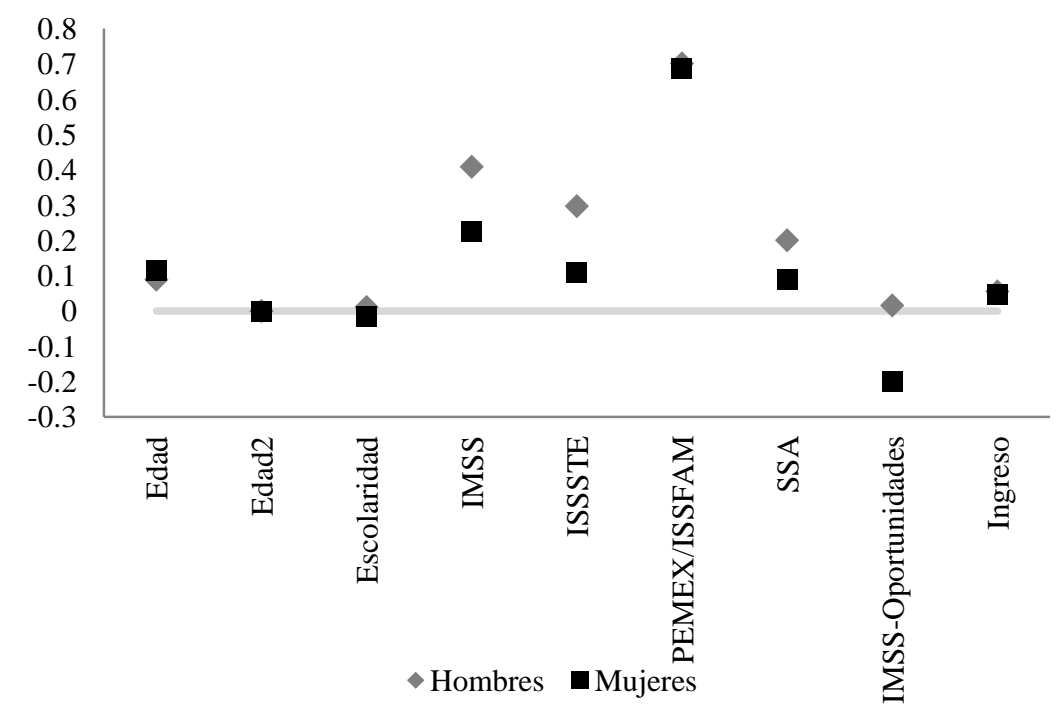

Fuente: Elaboración propia con datos de la ENSANUT 2012 (INSP, 2012) 


\section{Gráfica 5}

Probabilidad de factores de riesgo

a) Tomar

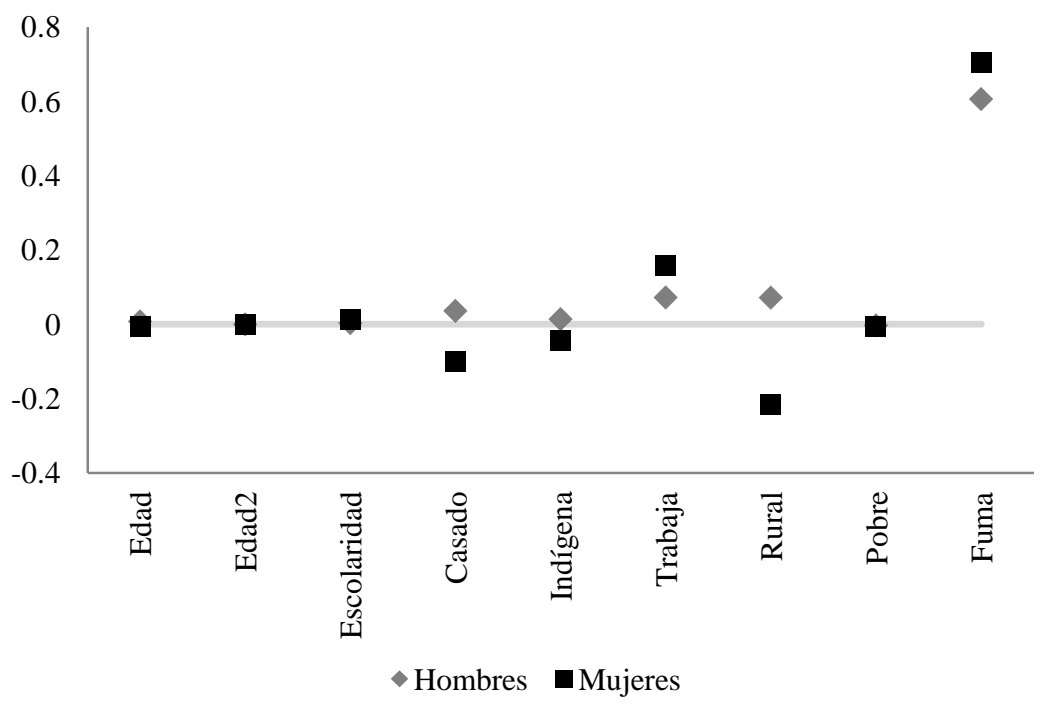

b) Fumar

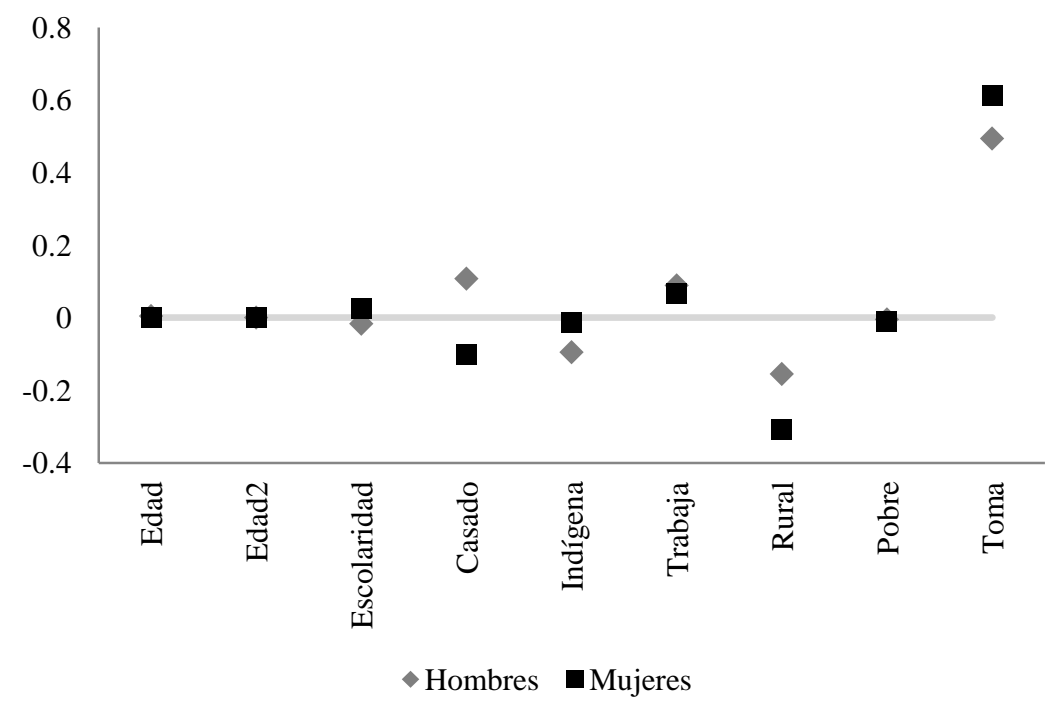

Fuente: Elaboración propia con datos de la ENSANUT 2012 (INSP, 2012) 


\section{Gráfica 6 \\ Probabilidad de tener obesidad}

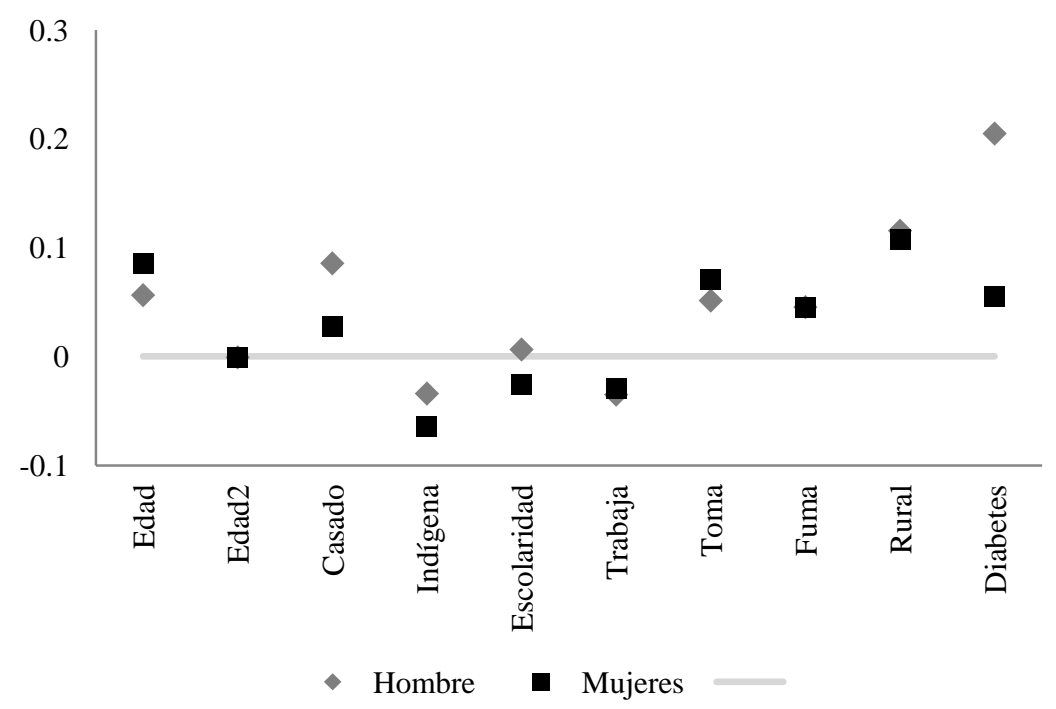

Fuente: Elaboración propia con datos de la ENSANUT 2012 (INSP, 2012)

Los efectos de los años de escolaridad en la probabilidad de que los individuos presenten una enfermedad crónica son, aunque significativos, muy pequeños y en su mayoría negativos, pero como ya se mencionó, son diferenciados para hombres y mujeres.

\subsection{Statu Quo}

Con la información de la ENSANUT 2012 del cuadro 1, y con los resultados de los modelos descritos en la sección anterior, se establece el punto de partida para llevar a cabo simulaciones.

Estos parámetros son los que pueden ajustarse para las simulaciones, es decir, podríamos jugar con escenarios donde el porcentaje de adultos obesos cae por alguna política pública, y observar cómo cambia el gasto en salud debido a esta disminución, o asumir que existe la cobertura universal y que todos los enfermos de estas tres enfermedades serán atendidos, ¿cuánto costaría?

\subsection{Gasto público en salud}

A partir de los resultados de los modelos de probabilidad de enfermedad, tratamiento y el costo promedio de las enfermedades, así como la definición del statu quo, se obtiene el gasto público en el que incurre el gobierno a 
través de las instituciones que proveen servicios de salud para 2015. Esto es 17,900 mdp para diabetes; 52,600 mdp para hipertensión y; 13,000 mdp para depresión. En total, suman 83,500 millones de pesos, lo que representa alrededor del $17 \%$ del presupuesto asignado a la función salud del Presupuesto de Egresos de la Federación en 2015 (SHCP, 2014) y 22.3\% de la subfunción "Prestación de Servicios de Salud a la Persona", la cual incluye la atención de las enfermedades crónicas.

\subsection{Ejercicio de simulación}

Al contar con un punto de partida y los parámetros que podríamos modificar ante distintos escenarios, lo que se propone en este apartado es realizar una simulación donde todos los adultos que hayan sido diagnosticados con una de las tres enfermedades analizadas en este estudio recibieran tratamiento, es decir, que el $9.1 \%$ de diabéticos, $15.9 \%$ de hipertensos y $10.7 \%$ de depresivos fueran atendidos con consultas, medicinas y cualquier servicios médico que necesiten por parte de las instituciones de salud pública, suponiendo que se quisiera alcanzar una cobertura universal en salud proveída por el Estado. Lo anterior supondría un aumento en el gasto público, pero, ¿de cuánto? El resultado de los cálculos arroja que el gasto en diabetes aumentaría 10.6\%, el gasto en hipertensión $28 \%$ y $37 \%$ el gasto en depresión. En total, el presupuesto para solamente atender estas enfermedades debería aumentar $26 \%$, mientras que el gasto total de la función salud debería tener un incremento de $4.1 \%$, solamente en 2015. Sin embargo, lo que realmente ha pasado con el presupuesto destinado a salud desde 2013 es que presenta una tendencia a disminuir, en vez de aumentar (gráfica 7).

Ahora, supongamos que las políticas públicas de prevención a la obesidad dieran resultado y la proporción de población adulta con sobrepeso y obesidad caiga cinco puntos porcentuales (de $22 \%$ a $17 \%$ ). El resultado sería una caída en la proporción de hipertensos en un punto porcentual de 15.9 a $14.8 \%$, lo que conllevaría una reducción del gasto en hipertensión, atendiendo al $67.7 \%$ de los hipertensos (INSP, 2012); el gasto total en salud disminuiría $2.6 \%$.

\section{Limitaciones en la metodología}

Este trabajo no está exento de limitaciones metodológicas, que no han sido incorporadas porque cada una de ellas es un tema de investigación en sí mismo, y realizarlo nos alejaba del objetivo principal: la construcción de un modelo de microsimulación para evaluar distintos escenarios. En esta sección detallaremos algunos de esto temas: 


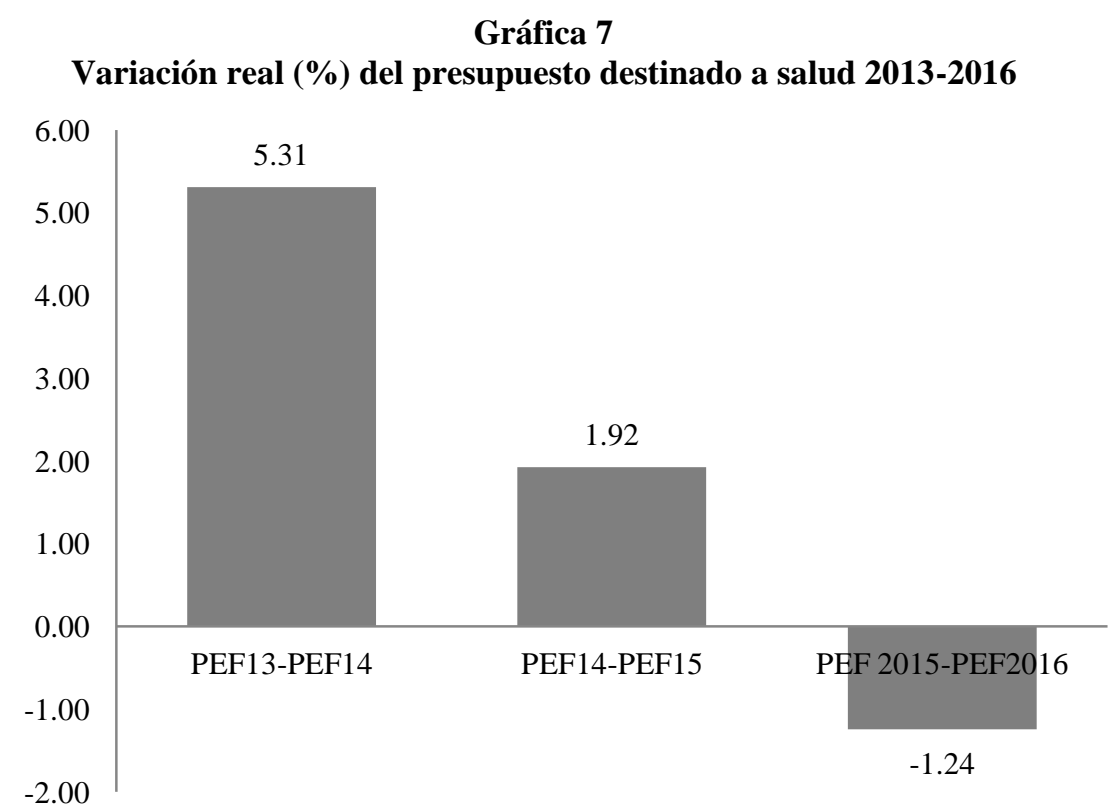

Fuente: Elaboración propia.

El primero se refiere a la disponibilidad de información sobre el diagnóstico y tratamiento de enfermedades crónicas o no transmisibles, así como su costo. Este trabajo se basó en información pública disponible, pero hace falta que las instituciones públicas sean más transparentes y constantes con respecto a la información que producen.

En segundo lugar, hablar de sostenibilidad a partir de tres enfermedades resulta corto, sin embargo, estas tres enfermedades son las que presentan mayor número de derechohabientes en IMSS e ISSSTE (más de 12 millones con diabetes y 12 millones con hipertensión). De hecho, la incidencia de diabetes ha crecido en $140 \%$ del año 2000 al año 2015, mientras que la hipertensión lo hizo en 92\% (IMSS, 2015). Un modelo integral no solo debería incorporar enfermedades crónicas, sino también las iniciativas preventivas y las enfermedades transmisibles, pero cada intervención presenta sus propias complicaciones.

El tercero es que este tipo de análisis tiene cierto grado de endogeneidad, como ya se mencionó anteriormente, dado que el diagnóstico y tratamiento de la diabetes depende de la disponibilidad y asistencia de las personas a acudir a una de las instituciones de salud, en otras palabras, es difícil saber si las personas que trabajan son las más sanas, o si debido a que trabajan, entonces, la probabilidad de enfermarse es menor. 
En cuarta instancia, el aumento en la cobertura no va acompañado de calidad en la provisión de servicios, de hecho, en este estudio se asume que todo lo demás permanece constante. Los costos no cambian, lo que indica que no existe cambio tecnológico, ni en la provisión de servicios, ni en la infraestructura. El análisis tampoco incorpora el alza en otro tipo de costos relacionados con el aumento en la cobertura. Aún con las limitaciones mencionadas, el análisis de este artículo es relevante por la metodología utilizada para generar el modelo de microsimulación, la cual es flexible y permite generar distintos escenarios. Además, muestra que la detección y el tratamiento de enfermedades crónicas aquí estudiadas es limitado por parte de las instituciones públicas, y que al aumentar la cobertura del tratamiento de todas las personas diagnosticadas con estas tres enfermedades, céteris páribus, el gasto en salud sería poco sostenible.

\section{Conclusiones}

Este artículo abre el debate para discutir sobre la cobertura efectiva y la cobertura universal en México, así como, para proveer de una herramienta de microsimulación que permita evaluar diferentes escenarios en cuanto a su costo financiero y poder hacer propuestas que descansen en metodologías rigurosas, de financiamiento sostenible principalmente, dado que se avecinan cambios demográficos y epidemiológicos que seguramente harán que los costos en salud se eleven, mientras que el presupuesto destinado a atender a la población disminuye.

Para alcanzar la cobertura efectiva universal en salud, deben realizarse cambios sobre la forma en la que actualmente se distribuyen los servicios de salud a la población. Dichos cambios deberán ir acompañados por el presupuesto correspondiente; sin embargo, saber a cuánto ascienden los gastos para atender a la población según sus necesidades de salud resulta complejo, debido a que los ciudadanos no planean cómo, ni cuándo enfermarse. Para ello, se construye un modelos de microsimulación basado en la probabilidad de que las personas se enfermen y reciban tratamiento por esa enfermedad, a fi de que junto con el costo promedio de tratamiento de tal enfermedad, se estime el gasto en el que se puede incurrir en distintos escenarios encaminados a proveer salud a toda la población.

Los resultados del modelaje del gasto público en salud arrojan que, en 2015, no se atendía al total de la población que presentaba enfermedades crónicas, como diabetes e hipertensión. El gasto asociado a este nivel de atención fue de $83,500 \mathrm{mdp}$, lo que representa el $17 \%$ del presupuesto total asignado a la función salud. Al hacer un ejercicio para cubrir al 100\% de los enfermos, quienes podríamos decir que son los adultos que necesitan de un tratamiento, 
el gasto total de la función salud debería aumentar 4.1\%. Lo anterior para el caso que se pretenda cubrir a toda la población con recursos públicos.

La cobertura efectiva universal puede construirse con un sistema único o fragmentado y, aunque un sistema único supone ser más eficiente, en el caso de México, también implica incurrir en costos políticos, por la existencia de sindicatos que probablemente se opondrían, pero también en costos referentes a los recursos, así como en la definición de paquetes únicos de salud para toda la población. Asimismo, determinar cuánto cuesta llegar a la meta universal es indispensable para saber cómo podría financiarse, si por impuestos generales o por aumentos en las cuotas de los afiliados, aunque debido a la gran proporción de la población que se encuentra en la informalidad, el cobro de cuotas se hace aún más complejo y costoso.

Los pasos a seguir en esta investigación son continuar modelando el gasto en salud, aumentando enfermedades crónicas como cáncer y enfermedades cardiovasculares, así como enfermedades del riñón. Paralelamente, se está desarrollando la posibilidad de proyectar el gasto en salud, con los parámetros obtenidos en el estatus quo en el futuro, para observar qué tan insostenible es el gasto en salud, incorporando la transición demográfica y la epidemiológica.

\section{Referencias}

[1] Lagomarsino, G., Garabrant, A., Adyas, A., Muga, R., \& Otoo, N. (2012). Moving towards universal health coverage: health insurance reforms in nine developing countries in Africa and Asia. Lancet, 380(9845), 933-943.

[2] CIEP. (2013). Estado actual de la Seguridad Social en México, 2013. Disponible en: http://ciep.mx/entrada-investigacion/estado-actual-de-la-seguridadsocial-en-mexico-2013/

[3] CONAPO. (Diciembre de 2015). Consejo Nacional de Población. Disponible en: http://www.conapo.gob.mx/es/CONAPO/Proyecciones_Datos

[4] CONEVAL. (28 de agosto de 2017). Medición de la Pobreza. Disponible en: http://www.coneval.org.mx/Medicion/MP/Paginas/Pobreza_2016.aspx

[5] Cotler, P., \& Rodríguez-Orregia, E. (2006). Microfinanzas y bienestar en el hogar en México. Escuela de Graduados en Admnistración Pública .

[6] Lymer, S., \& Brown, L. (2012). Developing a Dynamic Microsimualtion Model of the Australian Health System: A means to Explore Impacts on obesity over the Next 50 Years. Epideiology Research International , 1-14.

[7] Ávila-Burgos, L., Serván-Mori, E., Wirtz, V. J., Sosa-Rubí, S. G., \& SalinasRodríguez, A. (2013). Efectos del Seguro Popular sobre el gasto en salud en hogares mexicanos a diez años de su implementación. Salud pública de méxico , 55, S91-S99.

[8] Arredondo, A. (2014). Type 2 diabetes and health care costs in Latin. Medicine for Global Health , 12-136. 
[9] Banco Mundial. (septiembre de 2015). Cobertura universal de salud: Panorama general. Disponible en:

http://www.bancomundial.org/es/topic/universalhealthcoverage/overview

[10] Bolaños, R. d., Shigematsu, L. M., Ruíz, J. A., Márquez, S. A., \& Ávila, M. H. (2010). Costos directos de atención médica en pacientes con diabetes mellitus tipo 2 en México: análisis de microcosteo. Rev Panam Salud Publica, 28(6), 412-20.

[11] Bourgignon, F., \& Spadaro, A. (2006). Microsimulation as a tool for evaluating redistribution policies. Journal of Economic Inequality , 77-106.

[12] Bray, F., Jemal, A., Torre, L. A., Forman, D., \& Vineis, P. (2015). Long-term realism and cost-effectiveness: primary prevention in combatting cancer and associated inequalities worldwide. Journal of the National Cancer Institute, 107(12), djv273.

[13] Deaton, A. (2013). The Great Escape: health, wealth, and the origins of inequality. Princeton: Princeton University Press.

[14] Glassman, A., \& Zoloa, J. I. (2014). How Much Will Health Coverage Cost? Future Health Spending Scenarios in Brazil, Chile and Mexico. Disponible en: http://www.cgdev.org/sites/default/files/CGD-Working-Paper-382Glassman-Zoloa-future-health-spending-Latin-America.pdf

[15] Gómez, O., Sesma, S., Becerril, V. M., Knaul, F. M., Arreola, H., \& Frenk, J. (2011). Sistema de Salud en México. Cuernavaca, Morelos: Salud Pública de México.

[16] Guzmán, J. d., \& Vargas-Hernández, J. G. (2013). Modelo" plural-integral" de atención primaria a la salud enfocado a la satisfacción del usuario. Humanitas: Revista de Investigación , 10(10), 149-160.

[17] IHME. (2013). Global Burden Disease. Disponible en: http://vizhub.healthdata.org/irank/arrow.php

[18] IHME. (2013). Global Burden of Diseases, Injuries, and Risk Factors Study 2010. Disponible en:

http://www.healthdata.org/sites/default/files/files/country_profiles/GBD/ihm e_gbd_country_report_mexico.pdf

[19] IMSS. (2008). Grupos Relacionados con el Diagnóstico (GDR).

[20] IMSS. (2014). Informe al Ejecutivo Federal y al Congreso de la Unión sobre la situación financiera y los riesgos del Instituto 2013-2014. Instituto Mexicano del Seguro Social. Mexico, D.F.: Insituto Mexicano del Seguro Social.

[21] IMSS. (2015). Memoria estadísticas 2015. Disponible en: http://www.imss.gob.mx/conoce-al-imss/memoria-estadistica-2015

[22] IMSS. (28 de septiembre de 2012). Norma que establece las disposiciones generales para la aplicación de los grupos relacionados con el diagnóstico en el Instituto Mexicano del Seguro Social. Disponible en:

http://www.imss.gob.mx/sites/all/statics/pdf/manualesynormas/2000-001026_0.pdf

[23] INSP. (2012). Encuesta Nacional de Salud y Nutrición .

[24] Manuel, D. G., Tuna, M., Hennessy, D., Bennett, C., Okhmatovskaia, A., Finès, P., y otros. (May de 2014). Projections of preventable risks for cardiovascular disease in Canada to 2021: a microsimulation modelling approach. CMJA Open , (2), E94-E101.

[25] Musgrove, P. (1987). ¿ Cuanto mas vale prevenir que curar? reflexiones sobre la distribución de gastos en la atención médica. Economía , 10(19), 91-99. 
[26] OCDE Health Division. (2012). Toward a New Comprehensive International Health and Health Care Policy Desicion Support Tool. OECD Health Division.

[27] OECD. (2015). Health at a Glance 2015.

[28] OMENT. (2016). Últimas cifras de diabetes en México - ENSANUT MC 2016. Disponible en: http://oment.uanl.mx/ultimas-cifras-de-diabetes-en-mexicoensanut-mc-2016/

[29] OMENT. (2016). Uno de cada cuatro mexicanos padece hipertensión arterial ENSANUT 2016. Disponible en: http://oment.uanl.mx/uno-de-cada-4mexicanos-padece-hipertension-arterial-ensanut-2016/

[30] OMS. (2014). Cobertura Universal. Disponible en: http://www.who.int/mediacentre/factsheets/fs395/es/

[31] Paolillo, E., Russi, U., Cabrera, D., Martins, L., Scasso, A., Constantin, M., y otros. (2008). Grupos Relacionados por el Diagnóstico (GRD). Experiencia con IR-GRD en el Sanatorio Americano, sistema FEMI. Revista Médica del Uruguay, 24(4), 257-265.

[32] Pichon-Riviere, A., Augustovski, F., Bardach, A., Colantonio, L., \& Group3, f. t. (2011). Development and Validation of a Microsimulation Economic Model to Evaluate the Disease Burden Associated with Smoking and the CostEffectiveness of Tobacco Control Interventions in Latin America. Value in Health , 14, S51-S59.

[33] PiPP. (26 de julio de 2011). Caracterizacion de modelos sanitarios y sistemas sanitarios. Disponible en:

http://www.politicaspublicas.uncu.edu.ar/articulos/index/caracterizacion-demodelos-sanitarios-y-sistemas-sanitarios.

[34] Richard, P., Shin, P., Beeson, T., Burke, L. S., Wood, S. F., \& Rosenbaum, S. (2015). Quality and Cost of Diabetes Mellitus Care in. PLoS ONE 10(12): e0144075 .

[35] Rodríguez Bolaños, R. d., Reynales Shigematsu, L. M., Jiménez Ruiz, J. A., Juárez Márquez, S. A., \& Hernández Ávila, M. (2010). Costos directos de atención médica en pacientes con diabetes mellitus tipo 2 en México: análisis. Panam Salud Publica , 412-420.

[36] Rutter, C. M., Zaslavsky, A., \& Feuer, a. E. (2011). Dynamic microsimulation models for health outcomes: a review. Medical Decision Making , 31(1), 1018.

[37] SFP. (2015). Sectería de la Función Pública. Disponible en: http://www.gob.mx/sfp/acciones-y-programas/sistema-integral-deinformacion-de-padrones-de-programas-gubernamentales-siipp-g

[38] SHCP. (2012). Presupuesto de Egresos de la Federación 2013. Disponible en: http://www.apartados.hacienda.gob.mx/presupuesto/temas/pef/2013/

[39] SHCP. (2013). Presupuesto de Egresos de la Federación 2014. Disponible en: http://www.apartados.hacienda.gob.mx/presupuesto/temas/pef/2014/

[40] SHCP. (2014). Presupuesto de Egresos de la Federación 2015. Disponible en: http://www.apartados.hacienda.gob.mx/presupuesto/temas/pef/2015/

[41] SHCP. (2015). Presupuesto de Egresos de la Federación 2016. Disponible en: http://www.pef.hacienda.gob.mx/

[42] Soors, W., Devadasan, N., Durairaj, V., \& Criel, B. (2010). Community Health Insurance and Universal Coverage: Multiple paths, many rivers to cross. World Health Report. 
[43] Statistics Canada. (2015). Pohem (Population Health Model). Disponible en: http://www.statcan.gc.ca/eng/microsimulation/modgen/new/mods/pohem

[44] Urquieta-Salomón, J. E., \& Villarreal-Páez, H. J. (2015). Evalution on health coverage in Mexico: evidence of progress and challenges in the Mexican health system.

[45] USC. (2011). Royal Center for Health Policy Simulation. Disponible en: https://roybalhealthpolicy.usc.edu/about/

[46] Villarreal-Ríos, E., Campos Esparza, M., Galicia Rodríguez, L., Martínez González, L., Vargas Daza, E. R., Torres Labra, G., y otros. (2011). Costo anual per cápita en primer nivel de atención por género. Ciência \& Saúde Coletiva, 16(3) , 1961-1968.

[47] Wang, Y. C., McPherson, K., Marsh, T., Gortmaker, S. L., \& Brown, M. (2011). Health and economic burden of the projected obesity trends in the USA and the UK. The Lancet, 378(9793), 815-825. DOI: http://dx.doi.org/10.1016/S0140-6736(11)60814-3.

[48] World Bank. (septiembre de 2015). Going Universal: How 24 countries are implementing universal health coverage reforms from the bottom up. Disponible en:

http://www.worldbank.org/en/topic/universalhealthcoverage/publication/goi ng-universal-how-24-countries-are-implementing-universal-healthcoverage-reforms-from-bottom-up 
Apéndice A. Resultados de las regresiones

Tabla A1

Resultados de las regresiones de probabilidad de ser diagnosticado con diabetes - Hombres y mujeres

\begin{tabular}{|c|c|c|c|c|c|c|}
\hline & \multicolumn{6}{|c|}{ Diabetes } \\
\hline & \multicolumn{3}{|c|}{ Hombres } & \multicolumn{3}{|c|}{ Mujeres } \\
\hline & Coeficiente & $\begin{array}{l}\text { Desv. } \\
\text { Estándar }\end{array}$ & Valor Z & Coeficiente & $\begin{array}{l}\text { Desv. } \\
\text { Estándar }\end{array}$ & Valor Z \\
\hline Edad & $0.1427762 *$ & 0.000180 & 792.79 & $0.1120428 *$ & 0.000138 & 810.4 \\
\hline $\operatorname{Edad}^{\wedge} 2$ & $-0.0009972 *$ & 0.000002 & -644.16 & $-0.0007637^{*}$ & 0.000001 & -615.87 \\
\hline Casado & $0.1070021 *$ & 0.000962 & 111.2 & $-0.0765879 *$ & 0.000724 & -105.84 \\
\hline Indígena & $0.1608561 *$ & 0.000875 & 183.83 & $0.0446971^{*}$ & 0.000801 & 55.8 \\
\hline Escolaridad & $-0.0008756^{*}$ & 0.000081 & -10.76 & $-0.0368963^{*}$ & 0.000081 & -454.74 \\
\hline Trabaja & $-0.3444372 *$ & 0.000882 & -390.48 & $-0.1012595^{*}$ & 0.000782 & -129.42 \\
\hline Toma & $0.3369912 *$ & 0.003029 & 111.24 & $0.1587716^{*}$ & 0.003212 & 49.43 \\
\hline Fuma & $-0.035096^{*}$ & 0.000745 & -47.11 & $-0.0026451 *$ & 0.000840 & -3.15 \\
\hline Rural & $-0.2065761^{*}$ & 0.001076 & -192.06 & $-0.1855315^{*}$ & 0.000951 & -195.09 \\
\hline Toma*edad & $-0.0079853 *$ & 0.000055 & -145.65 & $-0.0052829 *$ & 0.000065 & -81.96 \\
\hline Obesidad & $0.185201 *$ & 0.000868 & 213.34 & $0.0283753^{*}$ & 0.000698 & 40.65 \\
\hline $\begin{array}{l}\text { Antecedentes } \\
\text { paternos }\end{array}$ & $0.4107123^{*}$ & 0.000937 & 438.49 & $0.3548848^{*}$ & 0.000851 & 417.14 \\
\hline $\begin{array}{l}\text { Antecedentes } \\
\text { maternos }\end{array}$ & $0.5211433 *$ & 0.000799 & 652.24 & $0.4873557 *$ & 0.000724 & 672.79 \\
\hline Constante & $-5.813646^{*}$ & 0.005315 & -1093.83 & $-4.595428 *$ & 0.003997 & -1149.64 \\
\hline
\end{tabular}

Notas: * Significativo al $95 \%$ de confianza 
Tabla A2

Resultados de las regresiones de probabilidad de ser diagnosticado con hipertensión - Hombres y mujeres

\begin{tabular}{|c|c|c|c|c|c|c|}
\hline & \multicolumn{6}{|c|}{ Hipertensión } \\
\hline & \multicolumn{3}{|c|}{ Hombres } & \multicolumn{3}{|c|}{ Mujeres } \\
\hline & Coeficiente & $\begin{array}{l}\text { Desv. } \\
\text { Estándar }\end{array}$ & Valor Z & Coeficiente & $\begin{array}{l}\text { Desv. } \\
\text { Estándar }\end{array}$ & Valor Z \\
\hline Edad & $0.0545418^{*}$ & 0.000121 & 449.66 & $0.0386759 *$ & 0.000095 & 409.34 \\
\hline $\operatorname{Edad}^{\wedge} 2$ & $-0.0002359 *$ & 0.000001 & -212.21 & $-0.0000757^{*}$ & 0.000001 & -84.2 \\
\hline Casado & $0.0675016^{*}$ & 0.000785 & 85.95 & $0.0191024 *$ & 0.000594 & 32.18 \\
\hline Indígena & $-0.0314724 *$ & 0.000785 & -40.12 & $0.0045847 *$ & 0.000660 & 6.95 \\
\hline Escolaridad & $0.0047717^{*}$ & 0.000071 & 67.71 & $-0.0245309 *$ & 0.000067 & -367.36 \\
\hline Trabaja & $-0.3025973^{*}$ & 0.000768 & -394.28 & $-0.1288286 *$ & 0.000617 & -208.75 \\
\hline Toma & $0.0101867 *$ & 0.002082 & 4.89 & $0.2172182 *$ & 0.002047 & 106.11 \\
\hline Fuma & $0.0200691^{*}$ & 0.000636 & 31.54 & $0.0487425 *$ & 0.000669 & 72.85 \\
\hline Rural & $-0.1779652 *$ & 0.000917 & -194.08 & $-0.1111831 *$ & 0.000753 & -147.7 \\
\hline Toma*edad & $-0.0006425^{*}$ & 0.000040 & -16.15 & $-0.0042514 *$ & 0.000045 & -94.43 \\
\hline Obesidad & $0.3326822 *$ & 0.000736 & 452.31 & $0.3197115^{*}$ & 0.000563 & 568.05 \\
\hline $\begin{array}{l}\text { Antecedentes } \\
\text { paternos }\end{array}$ & $0.4149722 *$ & 0.000813 & 510.48 & $0.3701577 *$ & 0.000676 & 547.46 \\
\hline $\begin{array}{l}\text { Antecedentes } \\
\text { maternos }\end{array}$ & $0.3836838 *$ & 0.000680 & 564.2 & $0.3883248^{*}$ & 0.000567 & 684.85 \\
\hline Constante & $-3.148794 *$ & 0.003317 & -949.24 & $-2.489906^{*}$ & 0.002590 & -961.51 \\
\hline
\end{tabular}

Notas: * Significativo al $95 \%$ de confianza 
Tabla A3

Resultados de las regresiones de probabilidad de ser diagnosticado con depresión - Hombres y mujeres

\begin{tabular}{|c|c|c|c|c|c|c|}
\hline & \multicolumn{6}{|c|}{ Hipertensión } \\
\hline & \multicolumn{3}{|c|}{ Hombres } & \multicolumn{3}{|c|}{ Mujeres } \\
\hline & Coeficiente & $\begin{array}{c}\text { Desviación } \\
\text { Estándar }\end{array}$ & Valor Z & Coeficiente & $\begin{array}{c}\text { Desviación } \\
\text { Estándar }\end{array}$ & Valor Z \\
\hline Edad & $0.0320433 *$ & 0.00014 & 223.05 & $0.0433274 *$ & 0.000097 & 449.19 \\
\hline $\operatorname{Edad}^{\wedge} 2$ & $-0.0003062 *$ & 0.00000 & -222.26 & $-0.0003993 *$ & 0.000001 & -414.22 \\
\hline Casado & $-0.2375075^{*}$ & 0.00088 & -270.52 & $-0.0993478 *$ & 0.000574 & -172.96 \\
\hline Indígena & $0.0767394 *$ & 0.00093 & 82.19 & $-0.1178595^{*}$ & 0.000679 & -173.6 \\
\hline Escolaridad & $-0.0018472 *$ & 0.00009 & -21.44 & $0.0103943^{*}$ & 0.000064 & 162.18 \\
\hline Trabaja & $-0.2092419 *$ & 0.00092 & -228.11 & $-0.0002427 *$ & 0.000585 & -0.41 \\
\hline Toma & $0.1801731 *$ & 0.00227 & 79.28 & $0.0454523 *$ & 0.001859 & 24.45 \\
\hline Fuma & $0.0318969 *$ & 0.00077 & 41.43 & $0.2117169 *$ & 0.000630 & 336.24 \\
\hline Rural & $-0.1653911 *$ & 0.00117 & -141.67 & $-0.187461 *$ & 0.000789 & -237.6 \\
\hline Toma*edad & $-0.0040679 *$ & 0.00005 & -84.98 & $0.000586^{*}$ & 0.000044 & 13.31 \\
\hline Obesidad & $-0.0788418 *$ & 0.00100 & -79.14 & $0.0339425^{*}$ & 0.000581 & 58.46 \\
\hline Diabetes & $0.0096267 *$ & 0.00124 & 7.78 & $0.1103399 *$ & 0.000856 & 128.85 \\
\hline Hipertensión & $0.6480771 *$ & 0.00096 & 672.99 & $0.4296444^{*}$ & 0.000662 & 649 \\
\hline Constante & $-2.242504 *$ & 0.00373 & -600.61 & $-2.228072 *$ & 0.002463 & -904.69 \\
\hline
\end{tabular}

Notas: * Significativo al $95 \%$ de confianza 
